\title{
INTRACRANIAL CYSTS
}

\section{ARACHNOID CYSTS}

A study of 67 childhood arachnoidal cysts is reported from the Service of Pediatric Neurology, Hospital "La Paza", Paseo de la Castellana, Madrid, Spain. Macrocephaly was the presenting symptom in 48 (72\%). Approximately $1 / 2$ were supratentorial and $1 / 2$ infratentorial in location; $15 \%$ were in the temporal fossa. Seizures were the presenting symptom in $7 \%$, mental retardation in $9 \%$, loss of developmental milestones in $3 \%$, and increased intracranial pressure in $5 \%$. The age at presentation was under 6 month in $37 \%$ and above 3 years in $24 \%$. Cranial asymmetry was present in $39 \%$ and aqueductal stenosis in $15 \%$. Small or medium sized cysts without clinical manifestations were not treated and were monitored once a year. Those cases associated with aqueductal stenosis were treated with cystoand/or ventriculoperitoneal shunting. (Pascual-Castroviejo I et al. Primary intracranial arachnoidal cysts. A study of 67 childhood cases. Child's Nerv Syst Sept 1991; 7:257-263.

COMMENT. Supratentorial arachnoidal cysts and particularly those involving the temporal lobe may be associated with perceptual and learning disabilities in children. The spontaneous disappearance of an arachnoid cyst in the middle intracranial fossa of a 21-year-old man is reported from the Departments of Neurosurgery, Neurology, Radiology and Psychology, University of Bergen, Haukeland Hospital, Bergen, Norway (Wester $\mathrm{K}$ et al. Neurology Sept 1991; 41:1524-6). This patient presented in early childhood with focal epileptic seizures, learning difficulties and a behavior disorder. An EEG at the age of 4 revealed abnormalities in the left temporal region and a $\mathrm{CT}$ at the age of 11 showed an arachnoid cyst in the left sylvian fissure. After a temporary increase in seizure activity at the age of 21 a second CT and an MRI showed that the cyst had disappeared entirely. A dichotic memory test initially showed an impairment of left temporal lobe function, but this improved when the test was repeated two months later. The authors consider that the brain tissue affected may regain function when the pressure from the cyst is relieved, even after years of anatomic distortion.

Migraine type headaches were the presenting symptom in 2 children with ADHD examined recently. EEG slowing in the left temporal area prompted examination with an MRI and revealed left medial temporal arachnoid cysts. The cysts showed no pressure effects and the headaches were treated conservatively by modification of the diet, with elimination of chocolate and caffeine containing drinks, and by the withdrawal of methylphenidate. Follow-up with repeat MRI studies was recommended (Millichap JG, unpublished case reports). 